\title{
Performance of Commercially Antibody-based assays for Covid-19 Detection
}

\author{
Maedeh Kojouri* \\ Department of Public Health and Infectious Diseases, Sapienza University of Rome, Italy \\ Department of Infectious Diseases, National Institute of Health, Italy
}

*Corresponding author: Maedeh Kojouri, Department of Public Health and Infectious Diseases, Sapienza University of Rome. Virology Laboratory, Department of Infectious Diseases, National Institute of Health, Rome, Italy Viale Regina Elena 299, 00161 Rome, Italy.

To Cite This Article: Maedeh Kojouri, Performance of Commercially Antibody-based assays for Covid-19 Detection. 2020 - 9(6). AJBSR.MS.ID.001453. DOI: 10.34297/AJBSR.2020.09.001453.

Received: 眥 July 13, 2020; Published: 阱 August 12, 2020

\begin{abstract}
The ongoing SARS-CoV-2 pandemic caused by novel coronavirus has become a threat to international public health. To tackle this pandemic reliable and rapid immunodiagnostic strategies are required. This study will briefly review the antibody diagnosis methods and recent clinical studies in this regard. The sensitivity and specificity after two weeks are considered to examine the late stages response of the disease. Among current studies on serology testing, 27 recent publications used commercial assays to diagnose IgM and IgG against SARS-CoV2 are discussed in this paper. A total of 54 kit-testing for antibodies, 20 are based on ELISA principles using serum or plasma samples with a sensitivity range of 55.6 $\%$ and $100 \%$ for IgG and between $77.3 \%$ and $82.7 \%$ for IgM. The specificity for both IgG and IgM ranges between $87.5 \%$ to $100 \%$. Twenty-seven kits are using lateral flow platforms and thirteen kits using luminescent immunoassays, including ten chemiluminescence immunoassay (CLIA) with sensitivity between $64 \%$ and $97 \%$ for both IgM and IgG and $75.6 \%$ to $100 \%$ for IgG. The specificity is reported between $85 \%$ to $100 \%$ and 90.9 to $100 \%$, respectively. Two Electrochemiluminescence Immunoassay (ECLIA) also showed high sensitivity and specificity for both antibodies. Antibody-based serology testing for SARS CoV-2 would be clinically important as a determination to return to work in society. Currently focusing on healthcare workers would be critical; however, there will be a significant need for serology-based testing to enable individuals to return to work or school safely in the overcoming months.
\end{abstract}

Key words: COVID-19; SARS-CoV-2; Antibody testing; Serological test

\section{Introduction}

The diagnosis of COVID-19 is mainly based on the polymerasechain-reaction (PCR) assay. Although PCR is high in sensitivity, there is a possibility of false-negative results. Numerous diagnosis immunoassays are developed to test COVID-19, including fluorescence assays, chemiluminescence immunoassays (CLIAs), enzyme-linked immunosorbent assays (ELISA), rapid antibody immunochromatographic test, and point-of-care test (POCT). Although there is a variation among test methods, they are mainly testing for the presence of two types of blood antibody, IgM and IgG or both. However, the timeline varies, and people respond differently to infection; these tests might detect antibodies by two or three weeks after symptom onset. These tests are not meant generally to active diagnosis infection but are able to identify prior infection. Many of these testing assays have not been validated yet, and their actual sensitivity and specificity are not fully understood.

\section{Materials and Methods}

\section{Search strategy}

PubMed, Embase, Medline, CAB Abstracts, ISI Web of Science, BIOSIS, LILACS, Cochrane library, CINAHL, AMED, National 
Research Register and In Process were searched applying the search terms "COVID-19", "SARS-CoV-2", "antibody testing", and "serological test", from December 2019 to June 2020. The studies using non-commercial kits and the ones which performed serology tests for less than two weeks have been excluded from this review.

\section{Data extraction}

From each publication, the following data were extracted: first author's last name, year of publication, the study name, follow up time, number of positive cases, number of control case, sensitivity and specificity of the kit for each Ig protein, method of testing and the kits manufacture company, country where the study was conducted.

\section{Diagnosis of COVID-19}

\section{Immunological and serological assays}

Analytical methods that use antibodies for detection are referred to as immunoassays. The reasonably specific binding of an antibody to their target makes immunoassays an effective method for the detection of analyzing complex biological matrices. Several types of immunoassays are used clinically in different designs, detection mechanisms, and how the assay reagents are combined with the sample.

\section{ELISA}

To perform the ELISA test, the wells of the plate are coated with the antigen of interest. By filling wells with the dilution of patient serum, the antibodies against the antigen will bind to the antigen fixed to the bottom of the walls in positive cases. Later the wells are washed out to remove unbound antibodies. Further, a solution of an animal antibody against human antibodies is added. The second antibody is covalently conjugated to an enzyme. The wells are washed out again this time to remove any unbound enzymeconjugated antibody. Finally, a colorogenic enzyme substrate solution is added. The interaction of the substrate with the enzyme on the second antibody generates visible color. The development of the color in the wells with a specific antibody can be seen with a naked eye or quantified with an electronic plate reader (Figure 1) [1].

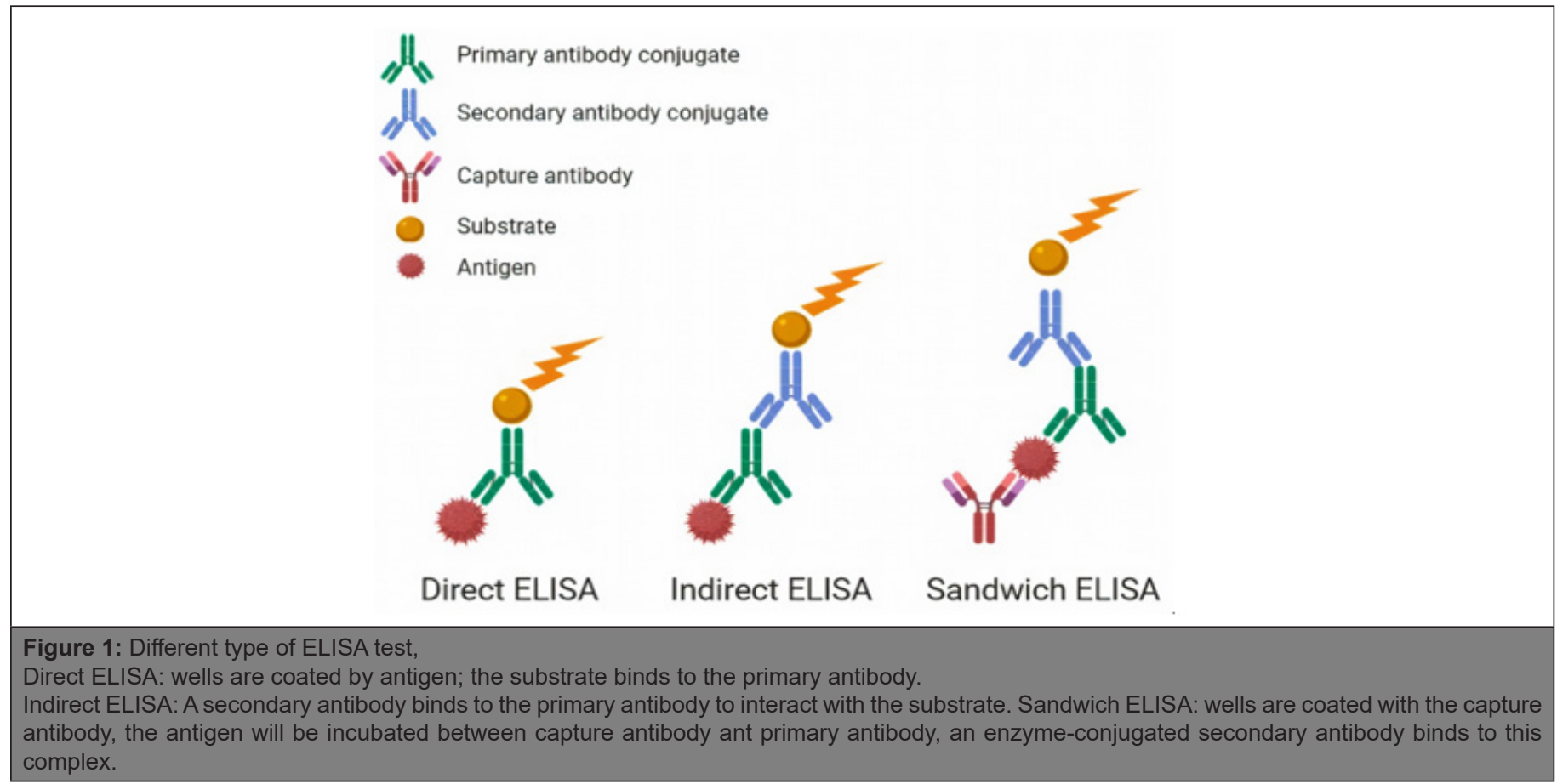

\section{Lateral flow assay}

In a lateral flow assay, the sample is added to the sample application pad at one end of the strip. The sample then migrates to the conjugate release pad, where a detection particle that has been conjugated to a biological component of the assay is held. The sample and the detection reagent migrate to the reaction membrane, where a second biological component of the assay has been immobilized in a test line. The detection reagent binds of the control further along the membrane to indicate that the assay has run successfully. The analysis is either captured at the test line or continues to migrate until it reaches the absorbent wicking pad at the end of the strip (Figure 2) [1].

\section{Luminescent analytical system}

The luminescent immunoassay is a technique capable of identifying specific antigenic molecules in the sample fluid, using the antibody and antigen labeled by an enzyme. There are several types of luminescent immunoassay, including chemiluminescent 
analytical system (CLIA) and electrochemiluminescence Immunoassay (ECLIA). In ECLIA, the species emit light as a result of electron transfer reaction generated by electrodes, while in CLIA, the light is the product of the chemical reaction.

\section{Plaque-reduction neutralization assay}

The presence of infection can also be detected using the plaque assay. Indicator cells that are permissive to infection are needed to perform the test. Sample contain viruses are added to previously cultured cells in tenfold serial dilution. The indicator cells are then washed and infected with diffract dilution of the virus. The overlayer suspension viscosity increases by using some agarose mix with a phosphate buffer saline, to ensure that each infected cell only infects its immediate neighbors. To make infection result more visible, the overlayer is peeled off, and the cells are fixed and stained using Coomassie brilliant blue dissolved in ethanol and acetic acid. After washing off all infected cells stained strongly blue in a compliant layer, however, any initial site of infection left a visible plaque, an area with no cells on the dish which appears white against the blue background. A range of different density plaques found in each plate is used to calculate platform unite (PFU) and expressed as a concentration pfu per milliliter (Figure 3) [3]

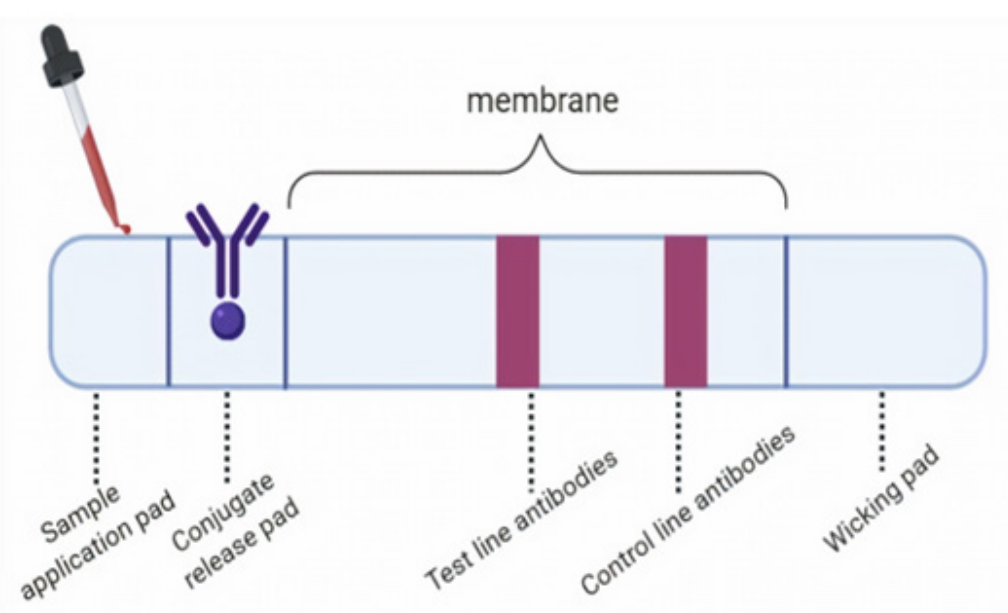

Figure 2: Latera Flow Assay

Patients sample including blood, serum, or plasma is added to the sample application pad, then the sample will migrate along the membrane for detection.

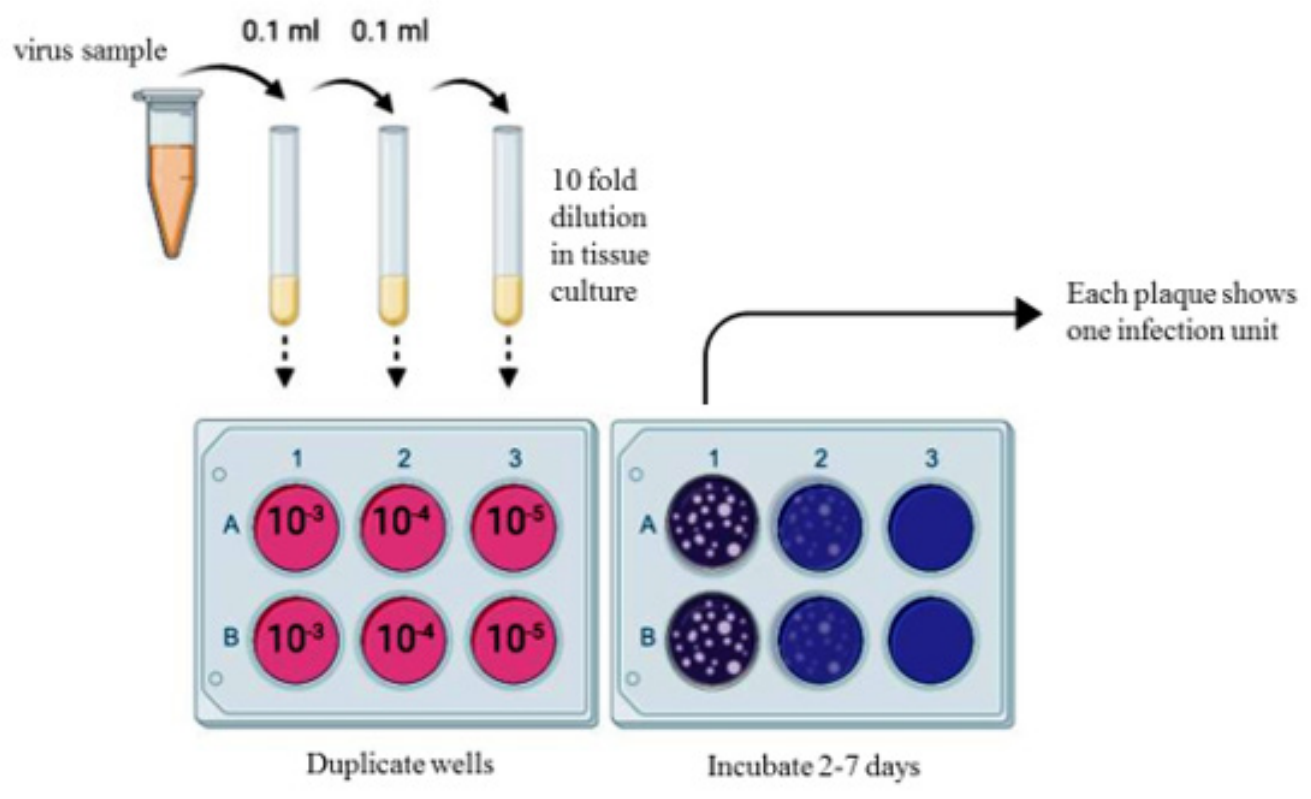

Figure 3: Neutralization Plaque Assay

Use to quantify the titer of neutralizing antibodies for a virus. 


\section{Result and Discussion}

In total, 27 studies including 2183 previously confirmed patient for COVID 19 were included in this review. Serological assays with the goal of finding IgM and IgG proteins are most effective within the few days after the onset of initial symptoms, and they are not advised as an acute phase diagnostic method. Several medical provisioner companies, scientific and research centers, have made great effort to develop the best diagnosis assays. A summary of recent studies using several commercial assays is presented in Table 1. Twenty ELISA kits are tested using a serum or plasma sample. Montesinos et al. investigated that Maglumi ${ }^{\mathrm{TM}} \operatorname{IgG} / \operatorname{IgM}$ tests show less sensitivity than the Euroimmun $\operatorname{IgG} / \operatorname{IgA}$ test $(84.4 \%$ versus $64.3 \%$ ). They also test three different lateral flow assays with sensitivity around (91\%-94\%) after 14 days of symptom onset [4].

Another clinical study by Xiang et al. reported that there is no significant difference between the sensitivity of ELISA and colloidal gold-immunochromatographic assay for both IgM and IgG testing [5]. Jan van et al. have tested seven rapid IgG/IgM tests and the Euroimmun IgA/IgG ELISA for antibodies against SARS-CoV-2. They found higher sensitivity for IgG in lateral flow assays compared to ELISA (92\% versus $89.5 \%$ ) after 14 days of symptoms onset [6]. Of the 20 ELISA based kits reported, the overall sensitivity range between $55.6 \%$ and $100 \%$ for IgG and between $77.3 \%$ and 82.7 $\%$ for IgM. The specificity for both IgG and IgM ranges between $87.5 \%$ to $100 \%$. Plebani et al. have tested Maglumi (Snibe), Liaison (Diasorin), iFlash (Yhlo), Euroimmun (Medizinische Labordiagnostika AG) and Wantai (Wantai Biological Pharmacy) assays and they reported diagnostic sensitivities and specificities above $93.8 \%$ and $85.9 \%$, respectively for all CLIA assays [7]. Thirteen luminescent immunoassays are tested, including ten chemiluminescence immunoassay (CLIA) with sensitivity between $64 \%$ and $97 \%$ for both IgM and IgG and $75.6 \%$ to $100 \%$ for IgG. The specificity is reported between $85 \%$ to $100 \%$ and 90.9 to $100 \%$, respectively [4, 7-11].

The colloidal gold-labeled immunochromatography assays have also been tested in three studies for both IgG and IgM. Presenting the sensitivity between $68.6 \%$ and $82.4 \%$ and the specificity between $96.2 \%$ and $100 \%$ [5,12,13]. Two Electrochemiluminescence Immunoassay (ECLIA) antibody also showed high sensitivity and specificity for both IgG and IgM antibodies [10,14-30].

\section{Conclusion}

As SARS-CoV-2 progresses over time, and the virus will be cleared from the immune system, it becomes more difficult to detect the viral RNA and viral protein using RT-PCR. However, the specific antibody produced against SARS-CoV-2 by the immune system can be detected using immunoassays. Reliable immunoassays tests can identify individuals who have already been infected and may not have been diagnosed at the time. The positive antibody test suggests that individuals may have gained immunity, or reinfection may not be as severe as the primary one. Identifying individuals who have already had infection is more important from a population health perspective than an individual's health prepositive. These individuals may help to identify the priority for vaccination or may be able to donate blood to critically sick patients who are fighting infection. During the pandemic COVID-19 reliable diagnostic test that targets both infected and recovered patients are needed to help combat the virus.

\section{Acknowledgment}

Maedeh Kojouri is funded by Virology Department at The Italian National Institute of Health (Istituto Superiore di Sanità, ISS).

\section{References}

1. K Shah, P Maghsoudlou (2016) Enzyme-linked immunosorbent assay (ELISA): The basics. Br J Hosp Med 77(7): C98-C101.

2. EB Bahadır, MK Sezgintürk (2016) Lateral flow assays: Principles, designs, and labels. TrAC - Trends Anal Chem 82: 286-306.

3. NMA Okba, MA Müller, W Li, C Wang, H Corine, et al. (2020) SARS-CoV-2 specific antibody responses in COVID-19 patients. 1-18.

4. I Montesinos, D Gruson, B Kabamba, H Dahma, S Van den, et al. (2020) Evaluation of two automated and three rapid lateral flow immunoassays for the detection of anti-SARS-CoV-2 antibodies. J Clin Virol 128: 104413.

5. J Xiang, M Yan, H Li, T Liu, C Lin, et al. (2020) Evaluation of EnzymeLinked Immunoassay and Colloidal Gold- Immunochromatographic Assay Kit for Detection of Novel Coronavirus (SARS-Cov-2) Causing an Outbreak of Pneumonia (COVID-19). MedRxiv.

6. J Van Elslande, E Houben, M Depypere, A Brackenier, S Desmet, et al. (2020) Diagnostic performance of 7 rapid IgG/IgM antibody tests and the Euroimmun IgA/IgG ELISA in COVID-19 patients. Clin Microbiol Infect 26(8): 1082-1087.

7. M Plebani, A Padoan, D Negrini, B Carpinteri, L Sciacovelli, et al. (2020) Diagnostic performances and thresholds: the key to harmonization in serological SARS-CoV-2 assays? Clin Chim Acta 509: 1-7.

8. A Padoan, C Cosma, L Sciacovelli, D Faggian, M Plebani, et al. (2020) Analytical performances of a chemiluminescence immunoassay for SARS-CoV-2 IgM/IgG and antibody kinetics. ClinChem Lab Med 58(7): 1081-1088.

9. M Infantino, V Grossi, B Lari, R Bambi, A Perri, et al. (2020) Diagnostic accuracy of an automated chemiluminescent immunoassay for antiSARS-CoV-2 IgM and IgG antibodies: an Italian experience. JMed Virol $1-5$.

10. N Kohmer, S Westhaus, C Rühl, S Ciesek, HF Rabenau, et al. (2020) Brief clinical evaluation of six high-throughput SARS-CoV-2 IgG antibody assays. J Clin Virol 129: 104480.

11. Y Jin, M Wang, Z Zuo, C Fan, F Ye, et al. (2020) Diagnostic value and dynamic variance of serum antibody in coronavirus disease 2019. Int J Infect Dis 94: 49-52.

12. B Shen, Y Zheng, X Zhang, W Zhang, D Wang, et al. (2020) Clinical evaluation of a rapid colloidal gold immunochromatography assay for SARS-Cov-2 IgM/IgG. Am J Transl Res 12 (14): 1348-1354.

13. Y Pan, X Li, G Yang, J Fan, Y Tang, et al. (2020) Serological immunochromatographic approach in diagnosis with SARS-CoV-2 infected COVID-19 patients. JInfect. 
14. M Egger, C Bundschuh, K Wiesinger, C Gabriel, M Clodi, et al. (2020) Comparison of the Elecsys ${ }^{\circledR}$ Anti-SARS-CoV-2 immunoassay with the EDITM enzyme linked immunosorbent assays for the detection of SARSCoV-2 antibodies in human plasma. Clin Chim Acta 18-21.

15. M Döhla, C Boesecke, B Schulte, CDiegmann, E Sib, et al. (2020) Rapid point-of-care testing for SARS-CoV-2 in a community screening setting shows low sensitivity, Public Health. 182: 170-172.

16. Y L Lee, CH Liao, P Y Liu, C YCheng, MY Chung, et al. (2020) Dynamics of anti-SARS-Cov-2 IgM and IgG antibodies among COVID-19 patients. JInfect 81(2): e55-e58.

17. L Spicuzza, A Montineri, R Manuele, C Crimi, M P Pistorio, et al. (2020) Reliability and usefulness of a rapid IgM-IgG antibody test for the diagnosis of SARS-CoV-2 infection: A preliminary report. J Infect 81(2): e53-e54.

18. WLiu, LLiu, GKou, YZheng, YDing, et al. (2020) Evaluation of Nucleocapsid and Spike Protein-based ELISAs for detecting antibodies against SARSCoV-2. JClin Microbiol 58(6): e00461-20.

19. FPérez García, R Pérez Tanoira, J Romanyk, T Arroyo, P Gómez Herruz et al. (2020) Alltest rapid lateral flow immunoassays is reliable in diagnosing SARS-CoV-2 infection from 14 days after symptom onset: A prospective single-center study. JClin Virol 129: 104473.

20. I Cassaniti, F Novazzi, F Giardina, F Salinaro, M Sachs, et al. (2020) Performance of VivaDiag COVID-19 IgM/IgG Rapid Test is inadequate for diagnosis of COVID-19 in acute patients referring to emergency room department. J Med Virol 1-4.

21. L Liu, W Liu, Y Zheng, X Jiang, G Kou, et al. (2020) A preliminary study on serological assay for severe acute respiratory syndrome coronavirus 2 (SARS-CoV-2) in 238 admitted hospital patients. Microbes Infect 2: 1-6.

22. R Lassaunière, AFrische, ZBHarboe, AC Nielsen, A Fomsgaard, et al. (2020) Evaluation of nine commercial SARS-CoV-2 immunoassays. MedRxiv.
23. T Hoffman, K Nissen, J Krambrich, B Rönnberg, D Akaberi, et al. (2020) Evaluation of a COVID-19 IgM and IgG rapid test; an efficient tool for assessment of past exposure to SARS-CoV-2, Infect EcolEpidemiol10(1): 1754538.

24. A JJääskeläinen, E Kekäläinen, H Kallio Kokko, L Mannonen, EKortela, et al. (2020) Evaluation of commercial and automated SARS-CoV-2 IgG and IgA ELISAs using coronavirus disease (COVID-19) patient samples, Euro Surveill. 25(18): 1-8.

25. K Imai, S Tabata, M Ikeda, S Noguchi, Y Kitagawa, et al. (2020) Clinical evaluation of an immunochromatographic IgM/IgG antibody assay and chest computed tomography for the diagnosis of COVID-19. JClinVirol128: 104393.

26. B Lou, T D Li, S F Zheng, Y Y Su, Z YLi, et al. (2020) Serology characteristics of SARS-CoV-2 infection since exposure and post symptom onset. Eur Respir J 19: 2000763

27. B Demey, N Daher, C François, J P Lanoix, G Duverlie, et al. (2020) Dynamic profile for the detection of anti-SARS-CoV-2 antibodies using four immunochromatographic assays. J Infect 81(2): e6-e10.

28. J Zhao, Q Yuan, H Wang, W Liu, X Liao, et al. (2020) Antibody responses to SARS-CoV-2 in patients of novel coronavirus disease 2019. Clin Infect Dis 1-22.

29.F Xiang, X Wang, X He, Z Peng, B Yang, et al. (2020) Ma, Antibody Detection and Dynamic Characteristics in Patients with COVID-19. Clin Infect Dis 1-23.

30. A Krüttgen, CG Cornelissen, M Dreher, M Hornef, M Imöhl, et al. (2020) Comparison of four new commercial serologic assays for determination of SARS-CoV-2 IgG. JClin Virol 128-104394. 